\title{
Philosophiques
}

\section{Pour une érotique philosophique}

\section{Benoît Proux}

Volume 30, numéro 2, automne 2003

URI : https://id.erudit.org/iderudit/008646ar

DOI : https://doi.org/10.7202/008646ar

Aller au sommaire du numéro

Éditeur(s)

Société de philosophie du Québec

ISSN

0316-2923 (imprimé)

1492-1391 (numérique)

Découvrir la revue

Citer cet article

Proux, B. (2003). Pour une érotique philosophique. Philosophiques, 30(2),

371-389. https://doi.org/10.7202/008646ar

\section{Résumé de l'article}

Il y a un silence de l'amour, et il y a un silence de la philosophie sur l'amour, qui la met au défi de trouver un nouveau langage, une nouvelle manière de penser et de sentir. Ce défi consiste pour la philosophie à correspondre pleinement à son essence, à être la philosophie. Relever ce défi est la tâche que se donne l'érotique. Ce sera une physique et une logique de la perception, une pensée de la pure surface, c'est-à-dire un discours de l'immanence. Ce sera l'épreuve de l'événement ontologique, au sens que Gilles Deleuze a su donner à ce concept.
Ce document est protégé par la loi sur le droit d'auteur. L'utilisation des services d'Érudit (y compris la reproduction) est assujettie à sa politique d'utilisation que vous pouvez consulter en ligne.

https://apropos.erudit.org/fr/usagers/politique-dutilisation/ 


\title{
Pour une érotique philosophique \\ BENOÎT PROUX \\ prouxb@aol.com
}

\begin{abstract}
RÉSUMÉ. - Il y a un silence de l'amour, et il y a un silence de la philosophie sur l'amour, qui la met au défi de trouver un nouveau langage, une nouvelle manière de penser et de sentir. Ce défi consiste pour la philosophie à correspondre pleinement à son essence, à être la philosophie. Relever ce défi est la tâche que se donne l'érotique. Ce sera une physique et une logique de la perception, une pensée de la pure surface, c'est-à-dire un discours de l'immanence. Ce sera l'épreuve de l'événement ontologique, au sens que Gilles Deleuze a su donner à ce concept.
\end{abstract}

SUMMARY. - There is a silence of love, and there is a silence of philosophy about love, which dares it to find a new language, a new way of thinking and feeling. For philosophy, this challenge consists in corresponding entirely to its essence, in being philosophy. Taking up this challenge is the task undertaken by erotic. This will be a physic and logic of perception, a thought of pure surface, which means a discourse of immanence. This will be the trial of the ontological event, according to the sense Gilles Deleuze was able to give to that concept.

Dans la mesure où la philosophie se désigne à elle-même comme amoureuse, penser l'amour lui apparaît comme une intime nécessité. Un savoir de l'amour peut-il résulter de l' "amour du savoir» ? Cette étymologie peut vouloir dire que la philosophie est savoir non encore devenu, puissance et attente de science. On peut aussi se demander si «aimer » ne serait pas une condition a priori de la possibilité de penser, la catégorie sans laquelle il n'y aurait pas de philosophie. En regard d'autres formes de la pensée, la philosophie serait la pensée qui aime, ou l'amour en tant qu'il pense et se pense. Nous nous proposons de réfléchir à la possibilité d'une philosophie de l'amour, qui manifesterait en soi-même, si l'hypothèse précédente s'avérait, l'essence de la philosophie.

Quel est l'objet d'une telle érotique? Qu'est-ce que tomber amoureux de quelqu'un? Il faut s'arrêter sur le fait, avant de considérer ce que vise ce sentiment, avant de savoir s'il y a lieu de considérer une telle visée. Car l'amour est un état avant que d'être une relation. Peut-être cesse-t-il dès l'instant où il devient une intention. Peut-être même n'est-il pas non plus un état, mais ce qui rend possible un certain état du corps.

En quel sens peut-il y avoir rapport nécessaire entre l'amour et la philosophie? Sur ce point, les philosophes font silence. Sans doute y a-t-il des philosophies du désir, de l'Éros intellectuel ou mystique, du sexe ou de l'instinct; il n'y a pas de philosophie du fait d'être amoureux. À moins que cette affection (c'est généralement ce qui se produit) ne soit appréhendée que comme état pathologique. Médecins, moralistes, théologiens, prêtres et 
devins prennent le relais, et c'est toute la clinique des passions qui se déroule. L'amour ne se distingue plus de la colère, de la tristesse, du plaisir, il perd sa singularité. Il apparaît alors comme l'autre de la philosophie. À quoi répond symétriquement le mépris quasi nécessaire qu'éprouve l'amoureux pour toute philosophie. Il faut prendre au sérieux chez Marivaux (par exemple dans La Seconde Surprise de l'amour) l'échec amoureux du philosophe, le ridicule, voire le scandale créés par ses propos lorsque les circonstances ou les rencontres le confrontent, pour lui-même ou chez les autres, à cette expérience. Soit par exemple le commentaire que Lacan donne du Banquet ${ }^{1}$ : Socrate n'affirme connaître que son ignorance, donnant ainsi naissance à la philosophie. Il y a cependant un domaine d'élection où il est fier de sa compétence: celui des choses de l'amour. À la fin du repas entre amis (à l'issue, on peut le noter, d'un concours de poésie, de parole), on en vient dans les vapeurs du vin à tenter de définir l'amour. Chacun des convives disserte. La thèse de Lacan (qui invoque ici l'autorité de Kojève) est que la relation de ces discours est pure ironie. Platon se moque de telles conceptions (l'amour est une harmonie, ou bien le manque d'une moitié perdue de l'Être, etc.) dont le ridicule est manifeste. Enfin, Socrate prend la parole. Lui, au moins, il sait, il va nous dire le vrai nom d'Éros. Et voilà que cette parole il la délaisse, qui plus est au profit d'une femme dont il doit être clair pour chacun que de sa bouche ne peuvent sortir que divagations (passer de l'amour des corps à l'amour des belles actions, des belles âmes, jusqu'au Beau qui n'est que Beau, tout cela c'est de l'amour qui n'est jamais de l'amour). Sur l'amour, Diotime délire, Socrate se tait, et Platon rit. D'autant qu'immédiatement après - admirable scénographie -, Alciabiade fait son entrée. Seulement, lui, il agit: il faut vaincre en effet la résistance de Socrate qui se dérobe à ses avances. Avec le bel Athénien, n'importe quel amoureux transi sait d'un savoir non conscient que la caresse, le baiser, la main saisie valent toujours mieux que la déclaration.

Quelle que soit la pertinence de cette lecture, il est certain qu'il y a un silence, une nescience consubstantiels à l'amour qui hypnotisent le philosophe et le plongent dans l'hébétude. C'est sa bêtise à lui, bêtise intelligente car nécessaire et révélatrice. Bizarrement, pourtant, l'amour n'est silencieux que pour la philosophie. Rien de plus bavard que lui par ailleurs. Peut-être même le langage dans sa totalité n'est-il qu'une longue, ininterrompue rhétorique amoureuse. Se produit là un curieux foisonnement de sens et de mots, une logorrhée continue rayonnant à partir d'un point de silence nescient, d'un point aveugle central. Avançons une hypothèse: ce silence de la philosophie sur l'amour, silence rendu plus profond encore par le contraste avec le feu d'artifice langagier qui éclate à partir de l'amour (littéra-

1. Jacques Lacan, Le Séminaire VIII, ensemble du chap. VI, "Le transfert», Paris, Seuil, 1991. 
ture, chant, poésie), serait une manière de ruse, une tactique pour se défaire précisément du langage et tenter de faire parler et paraître, dans des discours obliques, le corps en état d'amour. Ici surtout, la philosophie avance masquée. Elle fait silence sur l'amour, parce qu'il s'agit du point de néant de toute pensée, de l'impensable, sa tâche étant justement de s'efforcer de le penser en s'en éloignant sans cesse pour le dire sur le mode de la fuite, de l'échappement. Comme si l'amour exigeait, pour qu'il devienne possible d'en parler, exactement ce dont il a besoin pour advenir: une distance.

Parce que l'amour arrive à des corps et les modifie, l'érotique philosophique devra partir de la matérialité de l'amour, de sa physique. La méthode propre à cette érotique ne pourra être ni psychologique ni phénoménologique, mais logique et ontologique. Elle ne pourra être psychologique dans la mesure où l'on verra qu'on ne peut rapporter l'amour à une subjectivité déjà constituée, considérée indépendamment de son immanence à la Nature en général (temps et espace concrets). Elle ne pourra être phénoménologique, car elle contient une critique de l'intentionnalité comme rapport intériorisé du sujet à son objet: dans l'amour, il n'y a pas d'Autre, ni de dedans, mais un Dehors généralisé dont l'amour est un des modes. Si l'érotique est une logique, c'est en tant d'une part qu'elle détermine les conditions de possibilité (champ transcendantal) de l'«êtreamoureux ", d'autre part qu'elle se veut un discours systématique non pas transparent à soi-même (toute logique contient toujours de l'obscur), mais surtout présent à lui-même en toutes ses parties et en tous ses points, c'està-dire un discours de l'immanence.

De la même façon - mais c'est dire la même chose en un autre langage - l'amour est une expérience ontologique: l'épreuve d'un événement où remonte en surface l'Événément des événements: l’Être. Or une ontologie de la subjectivité (par exemple cartésienne ou husserlienne) semble incapable de saisir l'événement amoureux. L'érotique substitue donc à la dialectique de la profondeur et de l'intériorité une philosophie de la surface. Nous croyons ici que la manière dont Gilles Deleuze renonce aux catégories métaphysiques traditionnelles au profit d'une physique des devenirs et des intensités est seule capable d'affirmer l'amour. Le rapport nécessaire entre amour et pensée, c'est que la pensée de l'amour nous conduit à créer une nouvelle manière de penser.

Percevoir est la condition a priori pour tomber amoureux. Or un acte de perception est physique de part en part. À plusieurs reprises, Deleuze commente le premier chapitre de Matière et mémoire de Bergson ${ }^{2}$. Il n'y a pas p. 86-97.

2. Par exemple dans Cinéma I. L’Image-mouvement, Paris, Éditions de Minuit, 1983, 
d'un côté, dit Bergson, des choses (matière inconnaissable, étendue homogène) et de l'autre des représentations (idées, imaginations, désirs, intentions). Le tout de l'univers est toujours déjà donné, et les choses sont des images-mouvements: des images, parce que leur apparaître n'est rien d'autre que leur être; des mouvements, parce que leur être même est action et réaction des unes sur les autres, influence réciproque sur toutes leurs faces et en tous leurs points. Proximité de Bergson et de Leibniz: un oiseau, une étoile, une strate géologique se laissent traverser par la totalité des forces exercées par la totalité des autres réalités de l'univers, et c'est en cela que ce sont des choses. Un atome n'est pas un grain solipsiste, muré en soi, baigné de non-être; c'est une vibration commencée à l'autre bout du temps et de l'espace, et indéfiniment continuée au-delà d'elle-même. La matière est lumière. Ce qui veut dire aussi que le voir, le percevoir ne sont rien d'autre que des actions, et donc des distances, des événements diurnes, fragments du grand Midi. Les choses, les images-mouvements sont des perceptions en soi. Il n'y a pas de sujets de droit de la perception, mais seulement des occasions du voir: tout perçoit, parce que tout agit sur tout. L'œil est dans les choses, le miroir est partout. Et justement, l'amour est une perception qui a lieu dans une image-mouvement particulière qui s'appelle un corps: il est action et réaction physique: commotion matérielle, choc électrique dans les fibres sensitives - vérité du "coup de foudre»-, toucher des regards bien avant le contact des peaux. Ainsi le problème posé à l' "être-amoureux » (problème de la séduction) va-t-il être: comment trouver la bonne distance? Avant d'être une gymnastique, l'amour est une géométrie (figures, postures, positions), et même une machinique faisant intervenir les problèmes d'accord des temps et des espaces, c'est-à-dire les problèmes de vitesses.

Cette image particulière qu'est mon corps opère cependant une différenciation dans le tout infini et matériel de la perception, car elle constitue, comme dit Bergson, un centre d'indétermination. Mon corps, en effet (à l'instar de tout organisme vivant, bien qu'à des degrés divers), a cette capacité d'introduire un retard, un écart entre l'action qu'il subit et la réaction qu'il réfléchit sur les choses. C'est cet intervalle d'espace-temps, cette réponse différée, cette action seulement possible ou virtuelle qui forment ce que nous appelons pour nous la perception, uniquement définie donc par notre capacité d'agir plus ou moins efficacement, plus ou moins loin de notre corps. Entre la Grande Perception dans les choses et la petite perception dans les corps vivants, il n'y a qu'une différence de degré et non de nature. Bien plus, cette petite perception consiste à ne retenir dans les images-mouvements alentour que ce qui peut intéresser une action virtuelle de mon corps sur elles: elle dessine sur les choses un toucher potentiel, elle réfléchit sur elles les influences qui me concernent, elle laisse au contraire passer à travers le corps les ébranlements qui n'appellent en rien de sa part un mouvement. La petite perception consiste à obscurcir le monde pour se 
le rendre visible, à dresser l'écran noir du corps devant le flux rayonnant de la matière - la Nature est une cinématographie - pour qu'apparaisse le point de lumière, la réflexion sur la chose du mouvement que je reçois d'elle. Pour nos corps, il n'y a de perception possible que par un défaut, que dans un trou de la Grande Perception. La petite perception est la nuit du Grand Jour du monde.

Ainsi l'amour est-il rendu possible, comme le percevoir, par quelque chose de vide, quelque chose qui sert de plaque noire créatrice de distance. Ce qui à la fois rend possible le sentiment amoureux et constitue son véritable objet, ce n'est ni le beau, encore moins le bon, mais le charme. On ne tombe amoureux que si l'on tombe sous le charme. Ce qui est appelé ainsi n'est pas une qualité, mais plutôt l'absence d'une qualité (vide, écran obscur). Et cette absence n'est pas un manque, mais au contraire une consistance. La charme est ce qui distingue un individu, il est sa distinction. Il faut comprendre la "distinction " comme une différence en soi, c'est-àdire pensable seulement par rapport à elle-même, et pas du tout par rapport à une Identité-Genre première dont elle constituerait une espèce. On objectera que la beauté aussi ne peut pas se définir par rapport à une Essence antécédente ou Idée pure, qu'elle est toujours premièrement donnée dans le cas concret de beauté qui se présente ici et maintenant aux sens. Mais ce qui fait du charme autre chose que la beauté, c'est que, bien qu'il consiste, il n'a pas en lui-même d'identité assignable quelconque, harmonie de traits, proportion de qualités. Bizarrerie, étrangeté remarquable de quelqu'un: un défaut d'élocution, une manière de marcher, un éraillement dans le rire, le jais d'une chevelure comme un en-soi non rapportable à un sujet, comme un accident sans suppôt. Cette fêlure dans la plénitude indifférente de l'être-autre, cette absence attirent alors comme un vide. Le charme de quelqu'un est en lui ce qui donne le vertige, et donc retient l'attention, captive, envoûte, possède. Le charme n'est pas l'essence de l'être aimé, mais au contraire ce lieu du corps où il n'a pas d'essence. C'est sa faille, son point de démence, ce par quoi il nous échappe, ce par quoi il s'échappe à lui-même et se perd. Sa ligne de fuite. L'érotique devrait montrer que la perception du charme induit une topologie du corps de l'aimé, distribue l'espace de ce corps en unités non homogénéisables et non superposables, réorganise la vision du corps. Il faudrait indiquer par exemple comment le charme est nécessairement en rapport avec la visagéité, comment il fait de tout le corps une Figure, comme les corps dans la peinture de Bacon, qui, par le trait d'un contour, deviennent en tous leurs points quelque chose du visage, c'est-à-dire s'isolent du monde ${ }^{3}$. Le charme coupe du Monde, il sélectionne, il est élection, mais involontaire. C'est le point de flou déposé par la brosse ou le chiffon dans les Figures baconniennes; la ligne de

3. Voir le commentaire de Deleuze dans Francis Bacon. Logique de la sensation, Paris, Éditions de la Différence, 1981. 
démence, la case vide du corps. Aussi l'expérience du charme est-elle de l'essence de toute perception. Bergson l'indique dans Matière et mémoire: une perception est toujours une sélection; on ne voit pas tout, on souffre de ne pas tout voir. C'est la différence avec la représentation imageante une photographie, par exemple -, dont on souffre au contraire qu'elle soit pauvre, trop vite épuisée au regard: elle est déjà abstraite, complète par essence. Dans la perception "réelle", au contraire - et c'est ce qui fait d'elle toujours la perception d'un charme -, on perçoit ce que l'on voit ou sent, et en même temps qu'on ne voit ou ne sent pas tout. C'est l'écran noir, le fond virtuel, non actuel, mais absolument réel, sur lequel se découpe, se distingue ce qui est. Ce n'est pas du tout un au-delà, un fondement de la perception, un monde intelligible support du sensible; c'est au contraire inséparable du visible. Le fond n'est pas derrière, il est autour. Ce qu'on voit est toujours plus grand que nous (mais aussi toujours plus petit que nous). Il y a là un "à-sentir " insensible, qui pourtant ne peut être que senti, fond de toute perception: le Tout non totalisable, non dénombrable, plus grand ou plus petit, sur lequel est connecté le perçu partiel. Qu'on ne perçoive pas tout, cela aussi on le perçoit: telle est l'essence non thématisable du charme.

Nous disions que le charme est comme un vide qui attire. Il faut corriger et préciser: la perception du charme n'est pas encore une attirance, elle se situe avant elle. Au contraire, elle provoque une sorte d'arrêt ou d'horreur (timidité essentielle de l'amoureux transi, comme gelé par un froid), une impossibilité même provisoire d'aller vers. Le charme crée une réaction, pas une intention; une rétention, pas un mouvement. Ou plutôt le transport amoureux se fait sur place, mouvement tourbillonnant ou circulaire, intensif et non transitif. Le charme est une perception, une action sur le corps, mais en tant qu'il est l'indéfinissable (l'adjectif lui appartient presque en propre), le nescient, la ligne de fuite de l'être aimé. Le charme de l'autre - souffrance de l'amoureux - est ce qui le rend inquiétant, ce par quoi il ne répond pas à la préhension possible de mon corps sur le sien (le temps de l'amour, arythmique par rapport à celui du désir, c'est l'instant d'avant la saisie de la caresse). Le charme, c'est la frange nocturne sur la clarté de son corps et de son visage. Il faut bien distinguer cette horreur amoureuse de la fascination propre au désir. On pourra même se demander si le désir ne serait pas au contraire peur et désarmement de l'amour. Ce qui est éprouvé dans l'amour, ce n'est pas l'autre, mais une distance qui me sépare de lui et qui le sépare de lui. Tant il est vrai que tout devenir amoureux est une perception, et que toute perception, en tant qu'action et retard dans la réaction, déplie une distance justement (l'espace que dessine l'action réelle, mais non encore effectuée). Dans la perception du charme, on perçoit la distance par rapport à l'autre, mais aussi qu'il a une distance par rapport à lui-même (ligne de démence). La distance s'étend partout. Le charme est une distance orthogonale à la distance de la perception, mais 
qui ne constitue pas une autre dimension, une profondeur. Il s'agit plutôt de la répétition du même type de distance, dans d'autres directions: l'autre n'est pas orienté dans les mêmes directions que moi. L'amour est donc position de la distance et de la différence, d'abord parce qu'il est rendu possible par une étrangeté de l'aimé se formant devant l'amant, par une forme de négation de l'amant par l'aimé. «L'exquis contact des épidermes? Une embuscade tendue à l'autre pour que, renonçant à son regard et à sa liberté, il se fasse présence offerte. Une invite à la passivité. Une tentative d'empâter l'être désiré dans sa chair, pour qu'il ne s'échappe plus, et que je cesse, moi, de vivre sous son regard. Aussi tendre ou fervente soit-elle, la caresse est animée du désir de rendre l'Autre inoffensif, de le désarmer ${ }^{4}$.» Mais contrairement à ce qu'écrit Finkelkraut, c'est l'amour, et non pas du tout l'Autre, qu'il s'agit pour le désir ici de désarmer, précisément en se retournant vers l'Autre et en le désirant. La problématique du désir sera de supprimer l'amour (vérité de la vieille dualité amour-désir, mais pas en un sens moral), c'est-à-dire surtout d'empêcher la distance de se former. Le désir n'a de vérité que tactile ou "haptique». Le charme concerne prioritairement l'œil et l'oreille, l'odorat dans une moindre mesure. À ce moment précis, l'être amoureux n'éprouve aucun désir pour celui ou celle sous le charme de qui il est tombé amoureux. À ce moment précis, il est impuissant. Ce n'est pas un corps qu'il voit: il observe peureusement un Visage corporéifié, un Visage répandu sur tout le corps. Les analyses de Lévinas le disent bien. Si l'Autre se présente comme visage, c'est en tant qu'il échappe, qu'il est loin de moi dans sa proximité même, qu'il ne peut être défini ou thématisé. La nudité du visage, chez Lévinas, n'est-elle pas en rapport avec ce que nous appelions le charme? "Le visage est cette réalité par excellence où un être ne se présente pas par ses qualités ${ }^{5}$.» Aimer en l'autre non pas «une qualité différente de toutes les autres, mais la qualité même de la différence ${ }^{6} »$. Il nous semble pourtant que ce n'est pas le visage qui compte (il peut être dominé ou thématisé, circonscrit), mais la visagéité ou la visagéification du corps. Tout le corps rapporté au point de fuite, tout le corps organisé par le point de charme. Le charme n'a rien d'éthique, il est de la perception. Bien plus, l'attitude propre au devenir-amoureux sous le charme d'autrui n'est pas le face-à-face, mais le coup d'œil oblique, de côté, le regard en biais. Qu'il est difficile, qu'il est insupportable de regarder l'être aimé dans les yeux. Quand je regarde son visage, ce n'est pas lui que je vois, pas ses yeux, mais son regard, sous lequel je cille et vacille. Or le regard est la distance même. Je ne suis pas chosifié sous le regard d'autrui (verticalité) sans que s'opère auparavant une pétrification

4. Alain Finkielkraut, La Sagesse de l'amour, Paris, Gallimard, coll. «Folio Essais", 1984, p. 27.

5. Lévinas, Difficile Liberté, Paris, Albin Michel, 1963, p. 326.

6. Lévinas, Le Temps et l'autre, Montpellier, Fata Morgana, 1979, p. 14. 
de ce qui sépare tout mon corps de son visage (horizontalité). Cette distance n'est pas une grandeur. Elle est première par rapport à toute mesure possible. Elle ne se divise pas sans changer de nature. Elle est intensive avant de déplier une extension. En cela, l'amour est volonté de puissance: il n'est constitué, dans cette distance essentielle, que par une inégalité fondamentale (mon corps - le charme d'autrui), qui est le sens de l'inquiétude ou de l'horreur qui l'accompagnent: non pas attitude de négation, mais différence de potentiel créant l'intensité.

Nous parlions précédemment d'un transport amoureux qui se faisait sur place. Dans la mesure où la distance amoureuse engendre d'abord un espace, qui à son tour fait naître une temporalité (on l'a pressenti avec l'arythmie de l'être-amoureux par rapport au désir), tout amour comporte un problème de vitesse, c'est-à-dire de rapport entre un temps et un espace, tout amour comporte un devenir, tout amour est une vitesse. Mais cette vitesse a quelque chose de paradoxal. L'amour «ralentit» l'amoureux, lui confère une sorte d' "hébétude " indifférente qui le rend ridicule ou idiot aux yeux d'autrui. Il apparaît comme plongé dans un rêve éveillé, d'où le tire seulement ce qui a trait à l'objet de son émoi. Pourtant l'amoureux est aussi incroyablement «rapide»; il peut voir et sentir des signes trop mobiles, trop variables, trop fugaces pour être ordinairement perçus: ces signes innombrables émanent comme d'un foyer du corps visagéifié de l'être aimé. Comme l'animal, l'amoureux est l'être aux aguets. Ainsi chez Proust: "La manière anxieuse, exigeante que nous avons de regarder la personne que nous aimons, notre attente de la parole qui nous donnera ou nous ôtera l'espoir d'un rendez-vous pour le lendemain, et, jusqu'à ce que cette parole soit dite, notre imagination alternative, sinon simultanée, de la joie et du désespoir, tout cela rend notre attention en face de l'être aimé trop tremblante pour qu'elle puisse obtenir de lui une image bien nette. Peut-être aussi cette activité de tous les sens à la fois et qui essaye de connaître avec les regards seuls ce qui est au-delà d'eux, est-elle trop indulgente aux mille formes, à toutes les saveurs, aux mouvements de la personne vivante que d'habitude, quand nous n'aimons pas, nous immobilisons. Le modèle chéri, au contraire, bouge; on n'en a jamais que des photographies manquées ${ }^{7}$." Est décrit là exactement le flou d'une Figure, ou encore le défaut de focalisation, le défaut dans la mise au point, qui constituent le charme, la ligne de fuite. Proust nous ramène à Bergson et à Matière et mémoire. Tout est encore une conséquence de la nature de la perception, en tant que celle-ci est action et réaction des choses les unes sur les autres et en tous leurs points. Ce qui conduit à distinguer deux «mouvements" indissociablement liés, les deux conditions transcendantales du paradoxe de l'amoureux, et qui font du «devenir-amoureux » une véritable

7. À l'ombre des jeunes filles en fleurs, Paris, NRF, coll. «Pléiade», vol. I, p. 489-490; c'est nous qui soulignons. 
pensée. D’une part, se produit la sélection caractéristique de la petite perception dans les êtres vivants: tout sent et perçoit, mais simultanément et rapidement, à des vitesses inouïes. La vie, en obscurcissant les flux cosmiques, ralentit le monde. La vie (c'est-à-dire la pensée, les sensations), c'est de l'Être lent, de l'Être ralenti. De ce point de vue là, être conscient, c'est dormir. La conscience est comme une vacuole isolée par rapport à l'ensemble du plan de l'Être, une rupture temporaire de la plus grande partie des relations avec le reste de l'Univers, pour n'en conserver qu'une ou quelques-unes, devenues aspects privilégiés du monde, phénomènes. Ce ralentissement essentiel opéré par la pensée se fait sentiment et sensation corporelle totale dans l'état de perception amoureuse. Tout le corps devient maintenant surface d'enregistrement des perceptions triées, "sélectionnées ". Le corps de l'amoureux devient l'écran noir, le fond obscur sur lequel se détachent les actions et réactions en tant justement qu'elles se distinguent. Le charme agit, il y a quelque chose qui est aimable. La pensée amoureuse tourne à plein régime, il y a en elle de la machine: l'amoureux réfléchit, calcule, interprète, déchiffre. Mais en même temps (deuxième condition du paradoxe amoureux), se passe pour l'amour ce qui arrive à la pensée-perception: on sait qu'on ne perçoit pas tout, et c'est ce qui permet de percevoir; on sait que l'objet aimé s'échappe sans cesse, et c'est ce qui fait qu'on en tombe amoureux. Il y a un "à-sentir » insensible qui pourtant ne peut être que senti, il y a un "à-aimer» qui déborde infiniment toute capacité d'aimer, et qui constitue comme son champ transcendantal. Il y a autrement dit un écartèlement essentiel entre le perçu partiel lent (petite perception) et le tout du monde infiniment rapide (Grande Perception), entre la lenteur de l'amour (sélection des signes, des effets, des actions) et sa rapidité (connexion au Tout, qui est le charme comme ligne de fuite). La lenteur de la pensée ou de l'amour doit accueillir en elle le point à vitesse infinie qui est le Tout de l'être, qui manifeste le Tout de l'être. En cela, amour et pensée sont toujours arrachés à eux-mêmes. Il y a bien sûr des contre-feux: l'opinion, pour la pensée, tentera de ralentir cette vitesse du chaos de l'Être, mais aussi le désir, pour l'amour. La véritable pensée est donc conduite sans cesse au bégaiement, à ce bord dangereux où elle côtoie le silence et tente de le faire passer en elle-même; l'amour d'avant le désir est toujours confronté au risque, qu'il appelle pourtant, de devenir une sensibilité pure au Tout, un silence horrifié devant l'Être (la Grande Timidité). Il s'agit si l'on veut d'un affrontement avec la négativité, à condition de comprendre que celle-ci n'est pas un manque ontologique: elle est ce qui me dépasse et est plus grand que moi, non pas en nature, mais en quantité, en extension. Cette négativité consubstantielle à l'amour n'est en rien une négation de l'Autre, mais plutôt une négation de soi en tant que négation, négation de négation, affirmation de soi, répétition de soi, c'est-à-dire, donc, distance intérieure, écart de l'Être à soi-même. Tout cela est de la matière, et l'érotique dont nous parlons doit apparaître comme 
strictement matérialiste, donc comme une physique. Cette distance-écartèlement est première, immédiatement constitutive d'une étendue (extensio) dont le parcours (actions et réactions qui la traversent) forme une temporalité. L'amour comme vitesse est l'épreuve immédiate, le sentir de cette synthèse des dimensions du temps et de l'espace. La Distance amoureuse, loin d'être l'Autre de l'Être, est ce qui justement le constitue comme Être, autrement dit ce qui lui est intérieur (à condition de s'entendre sur le sens de ce terme: car cet intérieur de l'Être n'a rien à voir avec une intériorité, il est au contraire pur Dehors). L'amour est ainsi possible (nouvelle condition transcendantale) parce que l'Être s'aime lui-même et, ainsi, se trouve toujours à distance de lui-même. L'amour est une expérience à part dans la variété des sentiments et des émotions, parce qu'il est l'épreuve de l'Être par lui-même, le moment où l'Être, en dehors de toute conscience particulière, s'exprime à lui-même comme être. À la fois comme éloignement infini, mais aussi comme présence à soi infinie. Cette distance dans l'Être (substantialisation de l'étendue, ou plutôt l'étendue considérée comme l'attribut essentiel de la Substance, comme chez Spinoza), n'est pas un creux dans l'Être, un néant ou une néantisation; c'est une distance pleine, un perpétuel éloignement à soi de l'Être dans sa parfaite présence à lui-même en tous ses points. Cette distance «intérieure " à l'Être ne rompt en rien sa continuité essentielle. La distance de l'Être peut imiter la séparation; elle s'en distingue radicalement, cependant. L'Être ne sépare jamais. Il n'y a pas de néant, de vide (toutes les fois où nous avons dû employer de telles expressions, elles étaient incorrectes) qui rendraient possible le plein de l'Être. Il n'y a passage que du plein vers le plein, parce que le plein (Être) n'est autre que ce passage. En d'autres termes, la distance ne peut être appelée une négation qu'en tant qu'elle introduit de la différence, mais cette "négation" distanciante est pure affirmation. Une telle distance pleine peut donc être appelée surface. Par exemple, la distance pleine du corps vivant, c'est ses organes rassemblés et séparés par l'étendue de la peau, l'ensemble formant sa continuité, tout autant que sa distinction. C'est pour cette raison que nous disions que l'amour ne peut pas être compris dans une perspective phénoménologique: rien en lui n'est intentionnalité, c'està-dire visée par une intériorité d'un Extérieur relatif à elle (fausse sortie de soi). L'intentionnalité phénoménologique n'est pas ouverture au Dehors, mais constitutive d'une extériorité, c'est-à-dire assomption de celle-ci dans l'intériorité de la conscience: illusion d'immanence. La conscience dans ce cas ne se laisse en rien altérer par l'être de l'autre, puisque dans la néantisation qui constitue alors son essence, elle ne cesse de se mouler sur l'autre, d'en épouser les contours pour mieux rester ce qu'elle est. Ce n'est pas la conscience en tant que telle qui est ouverture aux choses; l'Être est immémorialement ouvert aux choses: une pierre à la pierre qui la jouxte, un mur à la mousse qui le recouvre. Il n'y a pas à s'ouvrir à l'extériorité, puisque celle-ci est toujours déjà là. Bien sûr, il y a de la conscience; mais 
celle-ci est fermeture aux choses. La transcendance de la conscience n'est donc qu'apparente. La conscience n'est pas un autre sens de l'Être, mais un ralentissement de la vitesse de l'Être. Il faut donc poser que l'amour ne saurait être un état de conscience. L'amour n'est pas visée de l'autre, car ce serait admettre une béance, une blessure néantisante au cœur de l'Être, qui renverrait celui-ci à autre chose que lui-même. L'amour n'est pas visée questionnante, puisqu'il est et ne peut être rien d'autre que pure affirmation de soi. Il n'est pas tension vers, ou explosion d'énergies contenues dans les abîmes du moi, mais expansion en surface. Il est l'Être sans cesse et sans fin répandu. Les notions d'intérieur et d'extérieur perdent toute signification; l'amour est étendue: comme elle, il est pure ouverture au Dehors; il est ce Dehors comme tel. D'ailleurs, d'où naît le concept d'intériorité ? De l'épreuve d'un sentiment dans le corps, d'une conscience des entrailles (battements accélérés du cœur, ventre noué, respiration oppressée). Mais ce qui se passe là est bien plutôt la remontée à la surface du corps, sur la peau, (tremblements, rougissements, transpiration) de ce qui se donnait comme indifférencié, ou plutôt encore virtuel, dans le corps. "Chez l'homme, le plus profond c'est la peau.» Tout le corps devient peau frémissante, les entrailles remontent, la surface s'enfonce et se répète, se multiplie dans toutes les directions. Pour cette raison, pas plus qu'il n'a de sujet (nous y reviendrons), l'amour n'a d'objet. Ce qui explique qu'il soit nescience et silence. De même qu'une volonté ne peut vouloir qu'elle-même, l'amour ne peut aimer que lui-même. D'où l'étonnement et l'incompréhension radicale de l'amant lorsque l'aimé lui dit éprouver aussi de l'amour pour lui. Qu'il aime aussi, en même temps qu'il est aimé, voilà qui ne peut jamais être thématisé. Au plus fort de la passion "partagée ", il y a une inégalité essentielle entre les amants. On est toujours seul à aimer, même si on le fait à deux, et nécessairement l'un des deux aime davantage: vérité ontologique avant que d'être psychologique. Il y a une essentielle solitude des étants sur le plan de l'Être, il y a un rapport à soi fondamental de la substance qui est un absolu non-rapport entre les modalités de la substance. Ainsi est-ce parce qu'ils ne sont définitivement rien d'autre qu'eux-mêmes, et donc absolument seuls, que les étants communiquent tous et simultanément sur le plan de l'Être. Cette solitude permet l'amour et la joie; elle constitue l'infinité du moi, ce au-delà de quoi il y a toujours quelque chose d'autre dans la continuité, c'est-à-dire dans ce qui nie la contiguité et pose l'isolement vital, celui de l'infiniment divisible que rien ne peut toucher, celui de la surface comme pur rapport à l'autre et pure extériorité à soi. L'amour ne peut s'expliquer comme blessure, vide ou manque; il est au contraire événement de la grande Santé de l'Être. La limite d'un étant, en effet, son altérité ne constituent ni sa périphérie ni son secret. La limite passe partout au milieu de l'étant, car celui-ci n'est rien d'autre que cet éternel devenir-autre qu'est la limite. Et ce passage continu d'une limite, c'est précisément une surface. Pourquoi en ce sens un être est- 
il sans limites, en vertu de quoi est-il de l'Être? Parce que les limites de son être, c'est son être même. L'Être n'est jamais limité par l'Être: voilà la consistance, le plan, la surface de l'Être. L'impénétrabilité d'autrui ne veut pas dire que je ne puisse le connaître, mais que je ne cesse pas d'apprendre à le connaître. Ma connaissance de lui est un devenir éternel, où nous devenons, non pas l'un l'autre, mais chacun entre l'un et l'autre, autre chose que nous-mêmes, en restant à jamais à distance l'un de l'autre. Autrui est impénétrable, non parce qu'il serait autre que moi, mais parce que comme moi, il est comme tout le monde, toujours autre que lui, parce qu'il est incessant et infini dévoilement, c'est-à-dire surface dépliée sans fond. Être amoureux, c'est affirmer l'identité infinie, que nous entendons comme présence à soi de la multiplicité en tant que multiplicité. Cette infinie présence à soi, cette affirmation positive traversant toutes les différences en tant qu'elles diffèrent, cette position des différences dans l'Être, position qui est l'Être et qui est la différence, c'est la surface. On objectera que le schème du volume eût convenu tout aussi bien. Si nous parlons de surface, plutôt que de volume de l'Être, c'est que l'essence de la surface est de n'avoir pas de profondeur. Et nous entendons par profondeur ce qui aurait une ou des dimensions d'une autre nature que celle de sa limite. Un volume, dont les différentes dimensions sont de même nature, mais de directions différentes, est donc bien une surface. On pourrait préciser: un enfoncement, une répétition de surfaces, une surface de surfaces, de même d'ailleurs qu'une ligne est un pli de surface et le point, une intersection de plis. Le devenir-amoureux, même dans sa part tragique, a donc cet aspect d'être une critique de toute forme de profondeur, d'être une superficialité, une légèreté essentielles. "Oh bonheur, j'ai accédé à ma propre surface par la haine et l'amour ${ }^{8}[\ldots]$ ", dit Nietzsche. Il n'y a pas lieu de placer la vérité de l'amour dans un ailleurs de l'amour, par exemple dans l'inconscient, autrement dit dans une profondeur. C'est pour cette raison qu'on peut oser écrire que le seul problème dont traite Sade est celui de l'amour. La quête des héros sadiens, quête dont l'échec ne cesse d'être affirmé, est celle de l'intérieur; d'où les découpages, les éventrements, les ouvertures, les dépliements des corps - impudeur suprême - dévoilés dans la vérité de leur intériorité: les entrailles. Mais l'entraille n'est pas aimable, elle n'est rien d'autre que du corps continué. L'amour n'est pas à l'intérieur, l'amour n'est pas un fond. Ainsi, puisque la recherche échoue, elle doit être répétitive, les personnages doivent être interchangeables, l'absence de fond nous renvoie sans cesse à l'infini de la surface. Sade, ou l'impossible nudité totale, puisque seule la peau (surface) est nue. 
Ce qui n'advient qu'en surface, c'est l'événement. Telle est l'essence (mais le langage de l'essence est-il encore adéquat?) de l' «être-amoureux». Non pas d'abord un vécu ou un état de chose, mais un acte, une vitesse, un "se passer ", permis par une distance (et donc des coordonnées topologiques) et engendrant une temporalité; toujours un pur devenir, jamais un état stable ou fixe, jamais un point d'arrêt. En cela, l'amour n'a rien de passionnel. La passion consiste dans un état de fixité, dans une permanence minérale qui chosifie la subjectivité, qui gèle et détermine la liberté en une essence. Mais l'état amoureux, quoiqu'il paraisse conférer lui aussi à l'amant une rigidité de cristal, est en réalité tout le contraire d'une telle stabilité pétrifiée. La "possession" amoureuse exprime le fait qu'il y a désubjectivation, dépersonnalisation de l'amoureux, et donc dissolution de son essence. L'amour est flux, qui emporte les scories du Moi et les disperse dans l'Être, et non pas, à rebours des autres passions, sculpture d'un Je à jamais coupé des choses et des modifications qu'elles opèrent. On est installé dans la haine ou l'état colérique ou la tristesse, mais on devient amoureux, sans cesse, et sans que l'on puisse assigner à ce devenir un quelconque état terminal, qui serait précisément la disparition de l'état amoureux. Reprenant une doctrine stoïcienne, Gilles Deleuze propose justement une théorie de l'événement fluide, qu'il oppose à la fixité des choses, et dont il nous semble que l'«être-amoureux" l'incarne de façon exemplaire. L'événement, par exemple, qu'est pour Alice le fait de grandir distribue deux aspects du réel: d'un côté, les corps et mélanges de corps, les états, les vécus, les substances et leurs accidents déterminés (le corps d'Alice, grand ou petit); de l'autre côté, ce qui arrive à ces corps ou ces mélanges de corps, mais ne se confond pas avec eux, c'est-à-dire les événements, qui ne peuvent être saisis que comme des actes et des effets de ces corps et mélanges de corps (grandir, ou bien diminuer, rougir, se promener, tomber amoureux...). Le lieu métaphysique d'une telle réalité, c'est la surface de l'Être. Deleuze cite Bréhier qui explique la pensée du Portique: "[Les stoïciens distinguent] radicalement, ce que personne n'avait fait avant eux, deux plans d'êtres: d'une part l'être profond et réel, la force; d'autre part le plan des faits, qui se jouent à la surface de l'être, et qui constituent une multiplicité sans fin d'êtres incorporels". " L'événement arrive en surface, et fait advenir la surface, parce qu'il est attribut logique, et non pas qualité ou propriété physique. Il est effet incorporel de corps qui sont causes pour d'autres corps. L'événement insiste, mais n'existe pas. Il n'est pas substantif ou adjectif, mais verbe, résultat d'actions et de passions. Deleuze nous entraîne encore plus loin que le stoïcisme: il ne s'agit pas seulement de distinguer dans l'Être sa surface incorporelle et sa profondeur réelle; il faut dire que l'Être en totalité est surface et événement sans

9. Bréhier, La Théorie des incorporels dans l'ancien stoïcisme, Paris, Vrin, 1928, p. 11-

13. 
profondeur, ou que la corporalité n'est rien d'autre que l'Être, en tant qu'elle est infinie remontée à la surface d'elle-même, pure présence à soi en son extériorité absolue à soi. Il ne faut surtout pas croire que l'événement en surface épuise, évapore l'Être: quoi de plus essentiel, de plus intime au corps que des événements comme grandir, rapetisser, être coupé, aimer? Qu'est-ce qu'un corps sinon le déroulement simultané de tous ces événements, leur déploiement dans l'espace? L'Être est tout entier «de l'autre côté du miroir». Citons le texte magnifique de Deleuze: "Là, les événements, dans leur différence radicale avec les choses, ne sont plus du tout cherchés en profondeur, mais à la surface, dans cette mince vapeur incorporelle qui s'échappe des corps, pellicule sans volume qui les entoure, miroir qui les réfléchit, échiquier qui les planifie. C'est en suivant la frontière, en longeant la surface, qu'on passe des corps à l'incorporel [...] [Alice] sait que les événements concernent d'autant plus les corps, les tranchent et les meurtrissent d'autant plus qu'ils en parcourent toute l'extension sans profondeur ${ }^{10}$.»

Le propre d'un tel devenir-événement est d'esquiver toujours le présent (il recueille ainsi en lui tous les paradoxes du devenir). "Alice grandissante ", le "grandir d'Alice» manifestent l'identité infinie des deux sens du temps à la fois, "du futur et du passé, de la veille et du lendemain, du plus et du moins, du trop et du pas assez, de l'actif et du passif, de la cause et de l'effet ${ }^{11}$ ». Le seul temps des états de choses, des corps, des substances, c'est le présent; et il y a un présent cosmique qui embrasse tous les présents des corps, et qui constitue à lui tout seul le Temps des corps. Mais la temporalité de l'événement est infinitive, illimitée division de l'instant entre passé et futur, et s'appelle alors Aion; ce que nous appelions précédemment vitesse: ce qui n'a pas d'arrêt ni de repos, ce qui jamais ne dort. " $[\ldots]$ car l'événement infiniment divisible est toujours les deux ensemble, éternellement ce qui vient de se passer et ce qui va se passer, mais jamais ce qui se passe ${ }^{12}[\ldots] »$

$\mathrm{Du}$ point de vue de la Nature, l'événement en surface révèle une nouvelle organisation de la relation causale. Les choses fixes, les sujets sont causes les uns pour les autres, et sont toujours causes. Il est de la nature de l'événement, et au premier chef de l'événement amoureux, d'être effet, d'arriver, d'advenir, de «tomber sur» (on «tombe amoureux»). La liaison des causes entre elles est appelée par les stoïciens: Destin. Mais les événements, n'étant qu'effets, ne peuvent entrer dans des relations de causalité les uns par rapport aux autres. C'est justement parce que dans les événements s'arrêtent l'enchaînement infini de la causalité, l'incessante sortie de soi que constitue la causalité, qu'ils recueillent en eux l'Être; devenirs purs,

10. Logique du sens, Paris, Éditions de Minuit, 1969, Paris, p. 19 et 20.

11. Ibid., p. 10.

12. Ibid., p. 17. 
ils sont pourtant le repos de l'Être. Entre eux existent néanmoins certains liens: il faut alors parler de "quasi-causalité». De ce caractère de l'événement découle aussi son impassibilité: n'étant ni corps ni mélange de corps, mais seulement l'effet des corps, et l'effet dans les corps, l'événement ne peut en lui-même être affecté. Impassibilité fondamentale du sentiment amoureux, que rien ni personne ne peut atteindre, qui ne diminue ou ne change qu'en cessant d'être, ce qui peut nous amener à penser à tort qu'il est comme toute passion, durcissement de la subjectivité. Car cette impassibilité est loin d'être immobile, elle est au contraire l'impassibilité même de tout devenir, l'impassibilité de la vie. L'amour est impassible en tant qu'il est continûment, en tant qu'il ne cesse d'être altération de ce à quoi il advient. Une altération jamais devenue, une altération jamais consommée, jamais résiliée dans la destruction ou son équivalent, l'unification. C'est ce que confirme l'autre aspect par lequel l'amour se présente essentiellement comme événement: son rapport intime au hasard. L' «êtreamoureux» ne peut être voulu ni prévu, bien sûr, il est toujours fortuit (le «coup de foudre»). Tout événement est fortuit, tout événement constitue le fortuit, puisqu'il est l'intersection d'au minimum deux séries indépendantes de causes (corps et états des choses, couteau et peau, charme et perception, action et réaction). L'intersection elle-même, le fait qu'il y ait intersection, n'est pourtant en rien cause d'autres effets. Ainsi l'événement suppose-t-il toujours une conjonction de séries, c'est-à-dire une multiplicité. Le hasard pose, affirme la pluralité, et la pluralité enveloppe toujours le hasard comme simultanéité des lignes causales. Même si on aime tout seul, on ne peut tomber amoureux sans qu'il y ait Deux. En réalité, beaucoup plus que deux; il y faut, comme dit Deleuze ${ }^{13}$, un agencement: on aime dans un certain contexte, dans un certain paysage, on est amoureux en compagnie de certains êtres (le ou les nécessaires confidents), et pas avec d'autres, etc. Le hasard implique donc la continuité. S'il n'y avait pas continuité, c'est-à-dire passage incessant dans l'autre, dans l'être-autre, s'il n'y avait ce fait d'être incessamment autre que soi, s'il n'y avait donc de l'extériorité (mais immanente, mais toujours en même temps soi), il n'y aurait pas de causes. L'existence de l'«être-amoureux» dénie la possibilité de la transcendance. Ce qui est dénié, c'est la position d'un pur autre qui n'aurait pas de communauté avec le soi, qui serait d'une autre nature, qui se dirait en un autre sens. C'est en même temps, donc, le refus de l'amour comme visée, intentionnalité, sortie de soi, dont on a montré le caractère illusoire. La transcendance est par essence ce qui sépare, ce qui nie de l'extérieur, constituant en tant que négation cet extérieur, lui donnant le nom d'Autre. Ce que nous rejetons au nom même de l'amour, c'est le sacrifice négatif qui introduit la différence en tant que fausse différence,

13. Par exemple dans Mille plateaux (avec Félix Guattari), Paris, Éditions de Minuit, 1980, p. 280 ou 317. 
différence rapportée à l'Un et non assumée en elle-même; il faut toujours un Christ sacrifié pour faire une transcendance. Le philosophe trouve donc un allié dans l'amoureux, car son effort est aussi de rejeter la séparation. La grande expérience amoureuse est celle de l'immanence, autre nom pour la surface. "Immanence » signifie que l'Être se dit d'une manière univoque, en un seul sens pour tous les étants. Pas de sens exemplaire, pas d'êtremodèle dont les choses seraient les copies approchantes, pas d'Autre du monde qui serait le fondement vrai du monde. Tous les étants communiquent sur la surface de l'Être, en ceci qu'ils sont, purement et absolument. Mais l'immanence (c'est exactement le paradoxe ontologique de l'amour) est simultanément et pour la même raison ce qui supporte la différence absolue entre les étants. «Une seule et même voix pour tout le multiple aux mille voies, un seul et même Océan pour toutes les gouttes, une seule clameur de l'Être pour tous les étants. À condition d'avoir atteint pour chaque étant, pour chaque goutte et dans chaque voie, l'état d'excès, c'està-dire la différence qui les déplace et les déguise, et les fait revenir, en tournant sur sa pointe mobile ${ }^{14}$." L'immanence est un nom, le nom: Monde, le monde qui n'est qu'un nom, qui n'exprime que l'illimité Infinitif de l'événement Être. C'est le néant des choses qui permet les choses. Ce qui veut dire au sens strict ceci: un Être commun, supérieur aux choses, une étoffe commune des choses n'existe pas, n'est pas. Ici la négation n'a que le sens d'une pure affirmation. Et d'autre part, l'immanence doit être sujet, jamais attribuée à autre chose qu'elle-même. Si nous disons de l'Être qu'il est immanent à lui-même, nous devons corriger, pour bien comprendre: l'Être, c'est l'immanence. Le sens de l'amour, c'est d'être justement un révélateur privilégié de l'Être-immanence, puisqu'il est cette distance pleine, cette surface, ce Un qui est l'affirmation de la différence absolue entre les amants. L'amour n'a pas de vérité — surtout pas éthique ou sociale sinon celle de son être-là : il est événement pur sur le plan de l'immanence. En conséquence, le fait amoureux est toujours négation de l'origine. L' « origine » et ses corollaires, famille et société, sont les premières figures de la transcendance. Platon avait déjà dit qu'Éros était fils d'Expédient et de Pauvreté, c'est-à-dire fils de personne, fils de rien. Il est même la destruction active de toute origine, contribuant ainsi au surgissement du plan d'immanence, toujours à construire. Pas d'amour qui ne soit par essence éclatement de la famille et puissance de révolution.

Orphelin par nature, l'amour est transi par une essentielle solitude, tellement essentielle qu'elle passe à l'intérieur du Moi amoureux et le disloque. Ou plutôt elle révèle l'illusion que constituait l'idée d'un Moi, en faisant advenir, en faisant arriver l'immanence même. Car si l'Être est, comment penser la relation entre les êtres? C'est la question du Sophiste.

14. Gilles Deleuze, Différence et répétition, Paris, Presses Universitaires de France, 1997, p. 389. 
Or justement, il n'y a pas de relation (autre ou néant de l'Être), mais seulement de l'Être, parce que celui-ci est le tout, l'affirmation des différences absolues: d'où l'essentielle, l'ontologique solitude. Pourquoi y a-t-il distance infinie du moi à l'autre? Parce que ce qui nous unit, l'Être, n'est pas de moi, mais me traverse, et lui aussi. Notre "parenté " sans origine vient justement de ce que nous ne sommes pas des sujets, mais que nous sommes ensemble dans l'extérieur, le Dehors. Désubjectivation, dépersonnalisation amoureuses. C'est en effet parce qu'il y a univocité de l'Être que ce dernier ne peut en rien, pas même comme représentation, être constitué par la conscience particulière. L'Être est soi-même et de part en part Conscience (la Grande Perception) comme présence à soi en tous ses points. Ainsi suis-je absolument seul dans sa continuité. Cette "ressemblance à tout ", ce "devenir comme tout le monde » qui me traversent sont la solitude active, celle qui permet de vivre. Il n'y a pas de sujet particulier, fermé sur soi, c'est-à-dire instance positionnelle d'extériorité. On l'a vu, tout sujet est toujours déjà à l'extérieur, moment de la grande subjectivité absolue. L'épreuve de la pensée, comme celle de l'amour, ne font pas de nous des sujets: en faisant passer à travers notre système nerveux (petits plis lovés au creux des grands Plis) le flux incroyablement lumineux du monde, au contraire elles nous défont, nous rendent aux multiplicités, dissolvent les fantômes de l'intériorité dans l'ouvert infini. Nous sommes matière et choses dans la matière des choses. C'est la conséquence de l'identité infinie de l'événement: la contestation de l'identité personnelle, la perte du nom propre. Le sujet, c'est ce à quoi il arrive des événements, à commencer par l'événement d'être, qui est le devenir des accidents et des qualités, c'està-dire des intensités parcourant l'extension de l'Être. Ce sujet est donc passif, "larvaire », c'est un résultat, et pas du tout quelque chose d'actif ou d'originant. La dépersonnalisation, à nouveau, n'est pas une négation. Au contraire, elle ouvre la possibilité de l'affirmation nécessaire du soi, c'està-dire de l'auto-affirmation de la vie qui nous traverse et est toujours plus grande que nous, comme devenir et passage. Voilà pourquoi il faut passer par l'abandon des unilatéralités. Dire que le sujet n'est pas, c'est dire qu'il n'y a pas de Un séparé, mais seulement des multiplicités, par quoi d'ailleurs la perception touche au monde, au Tout. Quitter l'unilatéralité de l'autre dans l'Être (mais c'est l'Être lui-même qui est ce départ), c'est faire que le courant passe, que les lignes se déploient. Ainsi s'expliquent d'une part l'absence d'objet, le «ratage» nécessaire de l'objet de l'amour, comme Marivaux l'a bien senti, et d'autre part le fait que l'amour n'est en rien un manque. Le manque suppose que l'on soit déjà soi, déjà constitué comme sujet: il est donc contradictoire en lui-même. L'amour n'aime rien, il aime, il est, il n'exige rien en retour, même pas lui-même, il n'est que l'expansion sans limite de l'Être. 
L'expérience amoureuse se manifeste en ceci qu'il n'est pas possible d'en parler adéquatement. Tous les discours amoureux ou sur l'amour sont à côté de l'amour; seul le discours immanent, le discours de l'Être lui-même peut parler de l'amour. Cette langue, qui est comme une langue étrangère, le philosophe veut apprendre à la parler. Mais alors il s'agit d'un langage qui ne cesse de lutter contre les mots et l'intériorité qu'ils constituent, d'un langage qui bégaie essentiellement, c'est-à-dire qui tente d'intégrer le silence à la pensée, et ce faisant l'expose au risque de l'annihilation, du rien. La philosophie de l'amour ne peut se concevoir que comme très haute poésie. Il y a en effet un rapport intime entre l'événement-effet et le sens ou la possibilité du langage en général. Car l'événement, n'étant pas corps mais surface du corps, incorporel attribué à la corporéité et la faisant être, ne peut être isolé que dans une proposition, plus précisément encore dans l'exprimé de la proposition. L'événement est le sens, le se passer impassible des étants. Il n'existe pas en dehors du langage. Mais il n'est pas non plus le langage et ses propositions: c'est à la chose, à l'étant qu'il s'attribue. Il n'est pas le prédicat (l'arbre est vert), il n'a donc pas de sujet grammatical ou logique; il est le verbe en son active neutralité ou impassibilité ou, mieux encore, il est ce que désigne le verbe en surface (verdoyer). L'événement-devenir est l'insaisissable dans les étants, ce qui ne peut être qu'exprimé dans les mots, renvoyant pourtant les mots à leur vacuité, les plaquant sur le corps de l'Être. Comme expérimentation propre à l'homme, à l'être du langage, l'amour apparaît encore plus essentiellement que tout autre événement comme la frontière des choses et des propositions, comme ce qui produit le sens à partir du silence des étants, comme la "case vide » permettant la genèse des séries du monde, comme le non-sens aléatoire circulant dans les séries du Tout. Nous comprenons mieux pourquoi l'amour peut être le silence par excellence, en même temps qu'il engendre toutes les significations (tout prend sens pour l'amoureux, tout exprime son amour) et fait jaillir les mots, surtout les plus bêtes, les plus simples. C'est la bêtise consubstantielle à l'amour (parallèle à l'essentielle bêtise du philosophe), l'idiotie de l'amoureux acéphale et aphasique, c'est la nescience et le silence du langage sur l'amour: l'impensé, véritable fond dans la pensée, qui ne peut être pensé qu'en arrachant la pensée à elle-même: l'événement. Surface des choses et genèse muette des propositions, l'événement amoureux est aussi le concept philosophique. Ce dernier peut être appelé abstrait, non pas en tant qu'il se séparerait de la matière, mais au contraire parce qu'en lui s'exprime la matière qui veut se séparer de la séparation, qui veut se séparer du langage, qui veut détruire le mot pour rendre possible à nouveau l'accès premier à ce qu'elle est en sa simplicité. "Abstrait» dans ce cas signifie bien "séparé », mais de la représentation, de l'idée, du mot. Le concept donc ne se découvre ni ne se contemple: il se crée. Seulement les concepts doivent être faits de mots. Et le langage 
effectivement est abstrait en lui-même. Les mots sont le silence des choses; celles-ci doivent imposer leur propre discours. L'Être est voix, il se dit, et se dit en un seul sens de tout ce dont il se dit, il est le seul Dit qui puisse se dire. La pensée philosophique se trouve donc confrontée à une forme d'impossibilité, coextensive en tous points à celle qu'éprouve l'amour, qui fait son vertige et parfois son ridicule. Le concept n'est concept, c'est-à-dire n'est concret, que lorsqu'il est pensée contre le langage et contre le sujet qui parle. La pensée ne pense jamais que contre elle-même: philosopher, c'est vraiment apprendre à mourir. Faire de la philosophie, comme être amoureux, c'est mourir à chaque instant, c'est-à-dire devenir le monde, et devenir dans le monde par-delà soi. C'est penser contre soi et tenter, dans un acte d'une folie évidente, d'intégrer la mort à la pensée comme surplus de vie, de faire de la pensée une éternité. Le philosophe, l'artiste, l'amoureux se débattent contre leur propre bégaiement, dans ce moment dangereux où ils veulent dire le silence. Ou plutôt, leur problème est de se taire — c'est le travail du concept, du percept, ou du devenir-amoureux — pour que s'élève enfin la voix de l'Être.

L'amour est donc l'universel vraiment concret; c'est une singularité au sens deleuzien: ce qui n'est ni particulier (c'est-à-dire défini dans son opposition à un universel) ni universel (il n'y a que des cas pratiques d'amour, ce dernier se présentant ainsi toujours sous la forme du problématique), parce qu'il n'y a pas dans l'Être des oppositions, mais seulement des différences (il y a..., il y a..., il y a...). L'amour est le particulier qui ne peut être le cas d'une généralité, qui n'est qu'en soi, qui n'a sa vérité qu'en soi. Il y a bien en lui quelque chose du Savoir absolu: il est auto-manifestation de l'Être ou Absolu (immanence), et surtout pas représentation de l'Absolu; il est même la critique de toute représentation, il est la nuit du savoir, non pas du tout à la manière de la théologie négative, mais comme trop-plein de l'affirmation de l'Être. En lui se dessine donc la possibilité d'une définition nouvelle de la philosophie. Celle-ci ne peut plus être la représentation médiatisée de l'Autre, forme vide et abstraite de l'Être. Aucun amoureux n'a jamais aimé un Autre: l'amour est présence pure à soi de l'être de l'aimé dans l'être de l'amant, amour de soi de l'Être. L'érotique rejette toute vision éthique de l'amour, qui reposerait précisément sur la transcendance, sur le pouvoir d'une telle forme vide: l' "Autre» (pouvoir et transcendance sont toujours des places vides). Seule subsiste la Différence, comprise comme pure affirmation de soi et de l'être: la négativité devient conjonction et connexion affirmatives des étants sur la surface de l'Être. 